\title{
TIM-3 in endometrial carcinomas: an immunotherapeutic target expressed by mismatch repair-deficient and intact cancers
}

\author{
Margaret Moore ${ }^{1} \cdot$ Kari L. Ring ${ }^{2} \cdot$ Anne M. Mills $\mathbb{B}^{3}$
}

Received: 29 November 2018 / Revised: 15 January 2019 / Accepted: 15 January 2019 / Published online: 29 March 2019

(c) United States \& Canadian Academy of Pathology 2019

\begin{abstract}
The checkpoint molecule TIM-3 is a target for emerging immunotherapies and has been identified on a variety of malignancies. Mismatch repair-deficient endometrial carcinomas have demonstrated durable responses to other checkpoint inhibitors due to high neoantigen loads and robust tumor-associated immune responses. However, little is known about TIM3 expression in this tumor type. Tumor-associated immune and tumoral expression of TIM-3 were evaluated by immunohistochemistry on 75 endometrial carcinomas [25 MLH1 promoter hypermethylated ( $M L H 1$-hypermethylated), 25 non-hypermethylated mismatch repair-deficient, and 25 mismatch repair-intact]. All cases showed at least focal immune staining, but moderate and robust immune cell expression were more often observed in mismatch repair-deficient vs intact cases [66 vs $12 \%, P=0.00002]$. While the majority (77\%) of endometrial cancers showed $\geq 1 \%$ tumoral TIM-3 expression, the $M L H 1$-hypermethylated subset was more likely to demonstrate $>5 \%$ tumoral staining when compared to both mismatch repair-intact and non-methylated mismatch repair-deficient cancers [64 vs. $28 \%$ and 32\%, respectively; $P=0.02$ and $P=$ 0.05]. Within the non-methylated mismatch repair-deficient subset, high-level expression was most often associated with MSH6 loss. Across mismatch repair subgroups, tumoral TIM-3 expression was more common among intermediate and highgrade vs. low-grade tumors using both the $1 \%(P=0.02)$ and $5 \%$ expression cut-offs $(P=0.02)$. In conclusion, tumoral TIM-3 expression is common in both mismatch repair-intact and deficient endometrial cancers, with particularly high levels of expression identified in the setting of MLH1-hypermethylation, MSH6 loss, and intermediate to high histologic grade. Although focal immune cell expression was seen in all tumors, robust expression was significantly more common in the context of mismatch repair deficiency. These data support a potential role for checkpoint inhibitors targeting TIM-3 in a subset of endometrial cancers, including some mismatch repair-intact tumors which are not currently considered immunotherapy candidates.
\end{abstract}

\section{Introduction}

Mismatch repair-deficient/microsatellite unstable tumors are increasingly considered as candidates for immunotherapy, and trials investigating agents targeting the PD-1/PD-L1 immune checkpoint pathway have shown efficacy in this

Anne M. Mills

amm7r@virginia.edu

1 University of Virginia School of Medicine, Charlottesville, VA, USA

2 University of Virginia Department of Pathology, Charlottesville, VA, USA

3 University of Virginia Department of Obstetrics and Gynecology, Division of Gynecologic Oncology, Charlottesville, VA, USA molecular context [1-5]. This therapeutic vulnerability is reflected in tumor histologies, immunohistochemical profiles, and molecular signatures: mismatch repair-deficient colorectal and endometrial carcinomas in particular demonstrate dense infiltrates of cytotoxic $\mathrm{T}$ cells, elevated neoantigen loads, and high-level expression of immune markers such as PD-L1 [6-13].

Despite these promising developments, response to targeted immunotherapy in solid tumors, including endometrial carcinomas, remains limited to a restricted group of patients [1, 14-18]. It is recognized that myriad immune modulatory molecules contribute to the tumor microenvironment, and that numerous mechanisms of immune escape may lead to incomplete response or resistance to monotherapy with immunotherapeutic agents $[9,14,19$ 21]. Alternative immunotherapeutic targets are therefore under investigation both as independent agents and in 
combination with other immunotherapies to optimize efficacy by more completely and precisely targeting the tumoral immune microenvironment $[15,22,23]$.

The immune checkpoint molecule $\mathrm{T}$ cell immunoglobulin and mucin-domain containing-3 (TIM-3) is one such potential target. TIM-3 suppresses immune activity using intracellular signaling mechanisms that are distinct from the PD-1/PD-L1 pathway [24-28]. TIM-3 is known to be expressed on Th1 CD4+ $\mathrm{T}$ helper cells, CD8+ cytotoxic T cells, regulatory $\mathrm{T}$ cells, and antigen-presenting cells $[26,27,29,30]$ and exerts immunosuppressive effects by promoting cytotoxic $\mathrm{T}$ cell exhaustion and increasing regulatory $\mathrm{T}$ cell activity $[24$, 29, 31]. TIM-3 expression has been demonstrated in the immune microenvironment of a wide array of neoplasms, including melanomas and gastric, urothelial, renal cell, cervical, and prostatic carcinomas [27, 32-35], and several early clinical trials of monoclonal antibodies against TIM-3 are underway in advanced solid malignancies [clinical trials.gov: NCT03489343, NCT02817633, NCT03680508, NCT03652077, NCT02608268].

The role of TIM-3 in endometrial carcinoma is still relatively unexplored. In particular, the impact of tumor grade in TIM-3 expression has not been well investigated, nor have prior studies compared TIM-3 expression in MLH1-hypermethylated cancers and non-methylated mismatch repair-deficient cancers, which may be of interest given that previous work has suggested that the immune milieu can vary with the molecular mechanism of mismatch repair-deficiency $[8,10,36]$. We herein investigate tumor cell and tumor-associated immune cell expression of TIM-3 in mismatch repair-intact and deficient endometrial carcinomas with attention to these variables.

\section{Materials and methods}

\section{Case selection}

This retrospective study was approved by the institutional review board of the University of Virginia. Whole sections of formalin-fixed, paraffin-embedded tissue from $25 \mathrm{MLHI}$ hypermethylated carcinomas, 25 non-methylated mismatch repair-deficient carcinomas, and 25 mismatch repair-intact carcinomas were assembled for evaluation. Clinical characteristics were abstracted from the electronic medical record. All cases were reviewed to confirm histologic typing and grade: endometrioid carcinomas were graded according to the three-tiered International Federation of Gynecology and Obstetrics (FIGO) system (FIGO $1=$ low-grade; FIGO $2=$ intermediate-grade; FIGO $3=$ high grade), and dedifferentiated carcinomas and carcinosarcomas were categorized as high-grade for statistical purposes. No serous endometrial cancers were included in the study due to the absence of this histotype among mismatch repair-deficient cancers in our files. Pathologic stage and clinical characteristics were abstracted from the electronic medical record.

\section{Mismatch repair status}

Mismatch repair deficiency was defined as the complete absence of nuclear expression of MLH1 (clone ES05, predilute; Leica Biosystems), PMS2 (clone MRQ-28Mab, predilute; Cell Marque), MSH2 (clone 25D12, predilute; Leica), and/or MSH6 (clone 44 Mab, predilute; Cell Marque) within tumor cells in the presence of intact control lymphocyte and stromal staining. These immunostains were performed in the University of Virginia Clinical Labs at the time of initial diagnosis as part of the institution's universal Lynch syndrome screening program. Additional testing for Lynch syndrome was performed according to the suggested algorithm outlined by Mills and Longacre [37, 38]. Non-methylated mismatch repair-deficient cases were defined as cases with total nuclear loss of expression for mismatch repair proteins MSH2, MSH6, and/or PMS2. Results from germline testing for Lynch syndrome were obtained from the patient's record when available; cases with discordant immunohistochemistry and germline testing results underwent microsatellite instability testing (Promega MSI Analysis Kit, Mayo Clinic Laboratories). MLH1-hypermethylated endometrial carcinomas were defined by loss of MLH1/PMS2 with confirmatory MLH1 promoter hypermethylation by pyrosequencing performed in the University of Virginia molecular laboratory (Epitech Bisulfite kit and MLH1 Primer Kit, cat \# 97002, Pyromark Q24, Qiagen).

\section{TIM-3 immunohistochemistry}

Immunohistochemical staining for TIM-3 (clone ab185703, dilution 1:400; abcam) was performed on whole sections of all 75 cases at the University of Virginia Biorepository and Tissue Research Facility. Immunostaining was scored manually at the microscope by two independent reviewers (M.M. and A.M.). Tumoral TIM-3 was classified as positive when membranous staining was present in $\geq 1 \%$ of tumor cells, a threshold based on previously proposed cutoffs for PD-1/PD-L1 [16, 17, 39], as there are no published criteria for TIM-3. Positive staining was further semi-quantitatively classified in the following subcategories: 1-5\%, 6-10\%, $11-25 \%, 26-50 \%$, and $>50 \%$; these thresholds were selected because they were included as cut-offs for multiple prior studies performed on PD-L1 [16, 17, 39]. Tumor-associated immune cell (lymphocyte and macrophage) staining for TIM3 was manually enumerated and averaged over 10 high power fields (HPF). Only immune cells in direct contact with tumor cells (intratumoral or directly peritumoral) were enumerated; cells in underlying stroma distant from the tumor were not 
counted. Tumors were then semi-quantitatively grouped as having absent, focal (1-20), moderate (20-40), or robust ( $>40)$ tumor-associated immune staining. Lastly, cases with a combined positive score (CPS) of $\geq 1$ were identified: this scoring system was recently developed for PD-L1, accounts for both tumoral and tumor-associated immune staining [positive tumor cells, lymphocytes, and macrophages)/ (total viable tumor cells) $\times 100$ ], and has been associated with predictive response to anti-PD-1 checkpoint inhibition in a variety of tumor types including gastric and gastroesophageal junctional carcinomas, small cell carcinomas, and recurrent cervical squamous carcinomas [40-42]. Tonsillar tissue was enlisted as the control, with scattered immune cell staining and squamous epithelial cell staining representing the expected pattern of expression.

\section{Statistics}

Descriptive statistics were calculated for variables of interest. Statistical analysis was performed using the two-tiered Fisher Exact Test for categorical variables and a one way ANOVA for continuous variables with SPSS statistics, version 25 (IBM, Armonk, NY).

\section{Results}

The 75 cancers were comprised of 67 endometrioid carcinomas (30 FIGO 1, 21 FIGO 2, 16 FIGO 3), six dedifferentiated carcinomas, and two carcinosarcomas. All 25 MLH1-hypermethylated cases were MLH1/PMS2-deficient and confirmed on PCR-based MLH1 hypermethylation testing. The 25 nonmethylated mismatch repair-deficient cases were comprised of eight cases with MSH6 loss, 12 cases with dual MSH2/6 loss, four cases with PMS2 loss, and one case with MLH1/ PMS2/MSH6 loss; this last case was MLH1-hypermethylation-negative. Patient consent for PCR-based germline testing was granted for 12 of 25 (48\%) cases, eight (67\%) of which showed confirmatory germline mutations. The four cases with discordant immunohistochemistry and germline testing results underwent microsatellite instability testing, and all were MSIhigh. Five of the six dedifferentiated carcinomas were nonmethylated mismatch repair-deficient cancers, while the remaining case was $M L H 1$-hypermethylated. One of the two carcinosarcomas was $M L H 1$-hypermethylated while the other was mismatch repair-intact. There were no significant differences in grade, stage, recurrence, or vital status across mismatch repair subgroups.

\section{Tumor-associated immune TIM-3 expression}

Tumor-associated immune cells demonstrated at least focal positivity for TIM-3 in all 75 cases reviewed (Table 1,
Table 1 Tumoral and tumor-associated immune TIM-3 expression by mismatch repair status, $n / N(\%)$

\begin{tabular}{|c|c|c|c|}
\hline & $\begin{array}{l}+ \text { TIM-3 }(\geq 1 \%) \text { in } \\
\text { tumor cells }\end{array}$ & +TIM-3 in TAIs & $\begin{array}{l}\text { TIM-3 CPS } \\
\geq 1\end{array}$ \\
\hline $\begin{array}{l}\text { MMR } \\
\text { deficient } \\
(N=50)\end{array}$ & $40 / 50(80 \%)$ & $50 / 50(100 \%)$ & $\begin{array}{l}50 / 50 \\
(100 \%)\end{array}$ \\
\hline \multirow[t]{7}{*}{$\begin{array}{l}\text { nm-MMRd } \\
(N=25)\end{array}$} & $19 / 25(76 \%)$ & $25 / 25(100 \%)$ & $\begin{array}{l}25 / 25 \\
(100 \%)\end{array}$ \\
\hline & $\begin{array}{l}\text { Extent of } \\
\text { expression: }\end{array}$ & $\begin{array}{l}\text { Extent of } \\
\text { expression: }\end{array}$ & \\
\hline & $1-5 \%: 11 / 25(44 \%)$ & $\begin{array}{l}\text { Focal: } 10 / 25 \\
(40 \%)\end{array}$ & \\
\hline & $6-10 \%: 5 / 25(20 \%)$ & $\begin{array}{l}\text { Moderate: } 8 / 25 \\
(32 \%)\end{array}$ & \\
\hline & $11-25 \%: 0 / 25(0 \%)$ & $\begin{array}{l}\text { Robust: } 7 / 25 \\
(28 \%)\end{array}$ & \\
\hline & $26-50 \%: 1 / 25(4 \%)$ & & \\
\hline & $>50 \%: 2 / 25(8 \%)$ & & \\
\hline \multirow[t]{7}{*}{$\begin{array}{l}\text { MLH1-hm } \\
(N=25)\end{array}$} & $21 / 25(84 \%)$ & $25 / 25(100 \%)$ & $\begin{array}{l}25 / 25 \\
(100 \%)\end{array}$ \\
\hline & $\begin{array}{l}\text { Extent of } \\
\text { expression: }\end{array}$ & $\begin{array}{l}\text { Extent of } \\
\text { expression: }\end{array}$ & \\
\hline & $1-5 \%: 5 / 25(20 \%)$ & Focal: 7/25 (28\%) & \\
\hline & $6-10 \%: 8 / 25(32 \%)$ & $\begin{array}{l}\text { Moderate: } 14 / 25 \\
(56 \%)\end{array}$ & \\
\hline & $11-25 \%: 4 / 25(16 \%)$ & $\begin{array}{l}\text { Robust: } 4 / 25 \\
(16 \%)\end{array}$ & \\
\hline & $26-50 \%: 3 / 25(12 \%)$ & & \\
\hline & $>50 \%: 1 / 25(4 \%)$ & & \\
\hline \multirow[t]{7}{*}{$\begin{array}{l}\text { MMR intact } \\
(N=25)\end{array}$} & $18 / 25(72 \%)$ & $25 / 25(100 \%)$ & $\begin{array}{l}25 / 25 \\
(100 \%)\end{array}$ \\
\hline & $\begin{array}{l}\text { Extent of } \\
\text { expression: }\end{array}$ & $\begin{array}{l}\text { Extent of } \\
\text { expression: }\end{array}$ & \\
\hline & $1-5 \%: 11 / 25(44 \%)$ & $\begin{array}{l}\text { Focal: } 22 / 25 \\
(88 \%)\end{array}$ & \\
\hline & $6-10 \%: 2 / 25(8 \%)$ & $\begin{array}{l}\text { Moderate: } 3 / 25 \\
(12 \%)\end{array}$ & \\
\hline & $11-25 \%: 2 / 25(8 \%)$ & Robust: $0 / 25(0 \%)$ & \\
\hline & $26-50 \%: 1 / 25(4 \%)$ & & \\
\hline & $>50 \%: 2 / 25(8 \%)$ & & \\
\hline
\end{tabular}

TAI tumor-associated immune, $C P S$ combined positive score, $M M R$ mismatch repair, MMRd mismatch repair-deficient, $h m$ hypermethylated, $\mathrm{nm}$ non-methylated

Figs. 1 and 2). However, there was a higher rate of moderate and robust TIM-3 immune cell staining in the mismatch repair-deficient $(33 / 50,66 \%)$ compared to mismatch repair-intact $(3 / 25,12 \%)$ tumors $(P=0.00002)$, and no intact cases demonstrated robust staining of tumoral immune cells. There was no significant difference between the degree of immune staining between the non-methylated mismatch repair-deficient and MLH1-hypermethylated subsets, and both had a relatively even distribution of focal, moderate, and robust expression. 

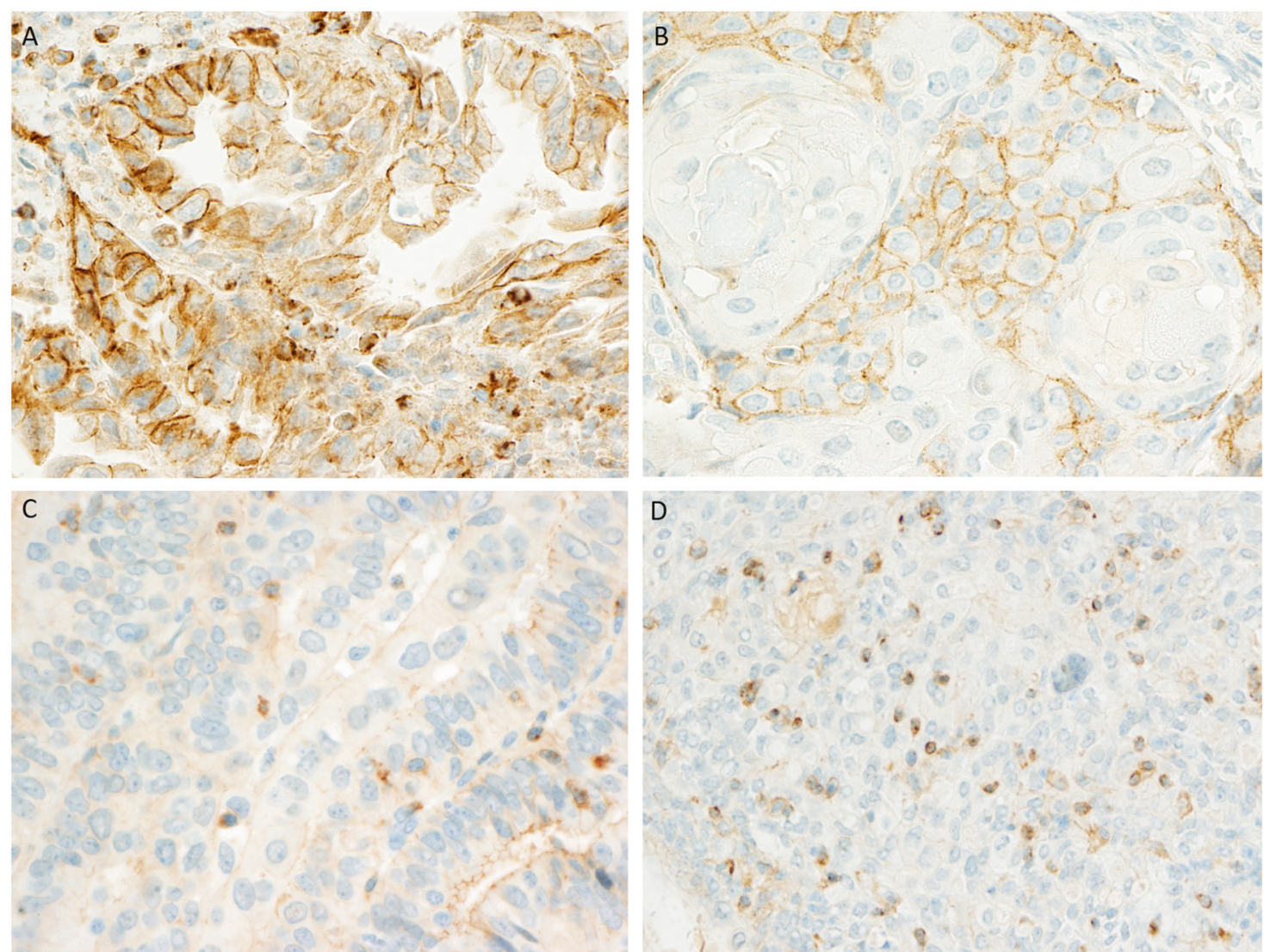

Fig. 1 Patterns of TIM-3 expression in endometrial carcinomas. TIM-3 was expressed in tumor cells (a, b) and in tumor-associated lymphocytes (c, d). Tumoral staining was considered positive when clear full or partial membranous expression was identified. Case a depicts a tumor with diffuse tumoral expression as well as scattered positive tumor-associated immune cells. Case b shows patchy tumoral

\section{Tumoral TIM-3 expression}

Tumoral expression of TIM-3 was demonstrated in the majority of both mismatch repair-intact and deficient endometrial carcinomas, with $72 \%$ (18/25) of intact tumors and $80 \%(40 / 50)$ of deficient tumors demonstrating at least $1 \%$ tumoral staining (Table 1, Fig. 1). Circumferential staining of small, scattered clusters of cells was the most common pattern, with most positive cases demonstrating expression in $<25 \%$ of tumor cells. However, five cases demonstrated 26-50\% expression and five more demonstrated $>50 \%$ positivity; these diffusely positive cases included tumors from all three mismatch repair subsets (Table 1). Although there was no significant difference between mismatch repair-intact and deficient tumors demonstrating positivity for TIM-3 using the $1 \%$ threshold for positivity (Table 1, Fig. 2), using a 5\% cut-off the MLH1-hypermethylated group was more often positive (16/ $25,64 \%)$ than both the mismatch repair-intact $(7 / 25,28 \%$; $P=0.02)$ and the non-methylated mismatch repair-deficient $(8 / 25,32 \% ; P=0.05)$ tumors. In contrast, there was no

positivity without a significant contribution of positive immune cells in this field (scattered positive tumor-associated lymphocytes were present elsewhere). Cases $\mathbf{c}$ and $\mathbf{d}$ show no tumoral expression (the apical blush depicted in some cells in case $\mathbf{c}$ was not considered positive), but focal (c) and robust (d) associated immune cell expression

significant difference in tumoral staining between nonmethylated mismatch repair-deficient and mismatch repairintact tumors using the 5\% threshold.

Tumoral TIM-3 staining was also evaluated in tandem with histologic grade and showed overall increased rates of positivity with increasing grade (Table 2, Figs. 3 and 4). A significantly higher number of intermediate- and high-grade carcinomas showed tumoral TIM-3 expression when compared to low-grade carcinomas using both the $1 \%(P=$ $0.02)$ and $5 \%(P=0.02)$ tumoral staining thresholds. However, this trend was not statistically significant for any of the mismatch repair subgroups when assessed in isolation. All six cases with dedifferentiated histology demonstrated tumoral expression of TIM-3. In addition, both carcinosarcomas showed tumoral expression of TIM-3. In both carcinosarcomas, TIM-3 expression was principally observed within the epithelial component with only limited staining in regions of mesenchymal differentiation (Fig. 5). Expression patterns were more variable within dedifferentiated carcinomas, with some cases showing staining primarily limited to well-formed glandular elements, some 

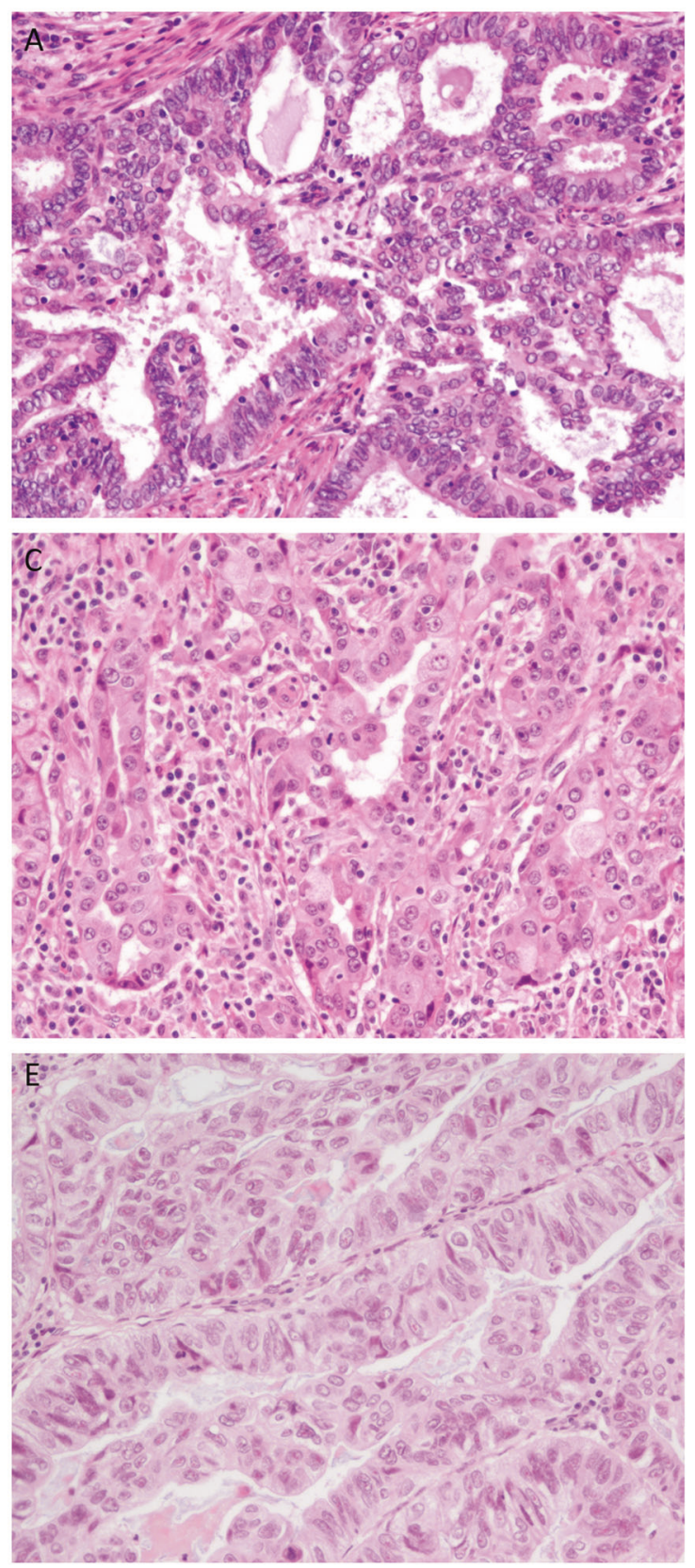

Fig. 2 Mismatch repair status and TIM-3. High levels of tumoral TIM3 expression were most often identified in endometrial cancers with MLH1-hypermethylation, like the case in (a, b). This case demonstrated tumoral staining in the majority of tumor cells as well as moderate associated immune cell expression. The MSH6-deficient

showing pockets of staining only in regions of dedifferentiation, and others showing a mixture throughout. (Fig. 5)

Of the non-methylated mismatch repair-deficient cases, the mismatch repair immunohistochemistry pattern most consistently associated with TIM-3 expression was loss of MSH6, with all eight tumors in this group demonstrating $\geq 1 \%$ tumoral staining, and two of the cancers demonstrating $>25 \%$ staining (Table 3). The sole case with deficient MSH6 and loss of MLH1/PMS2 was also TIM3-positive. Tumors
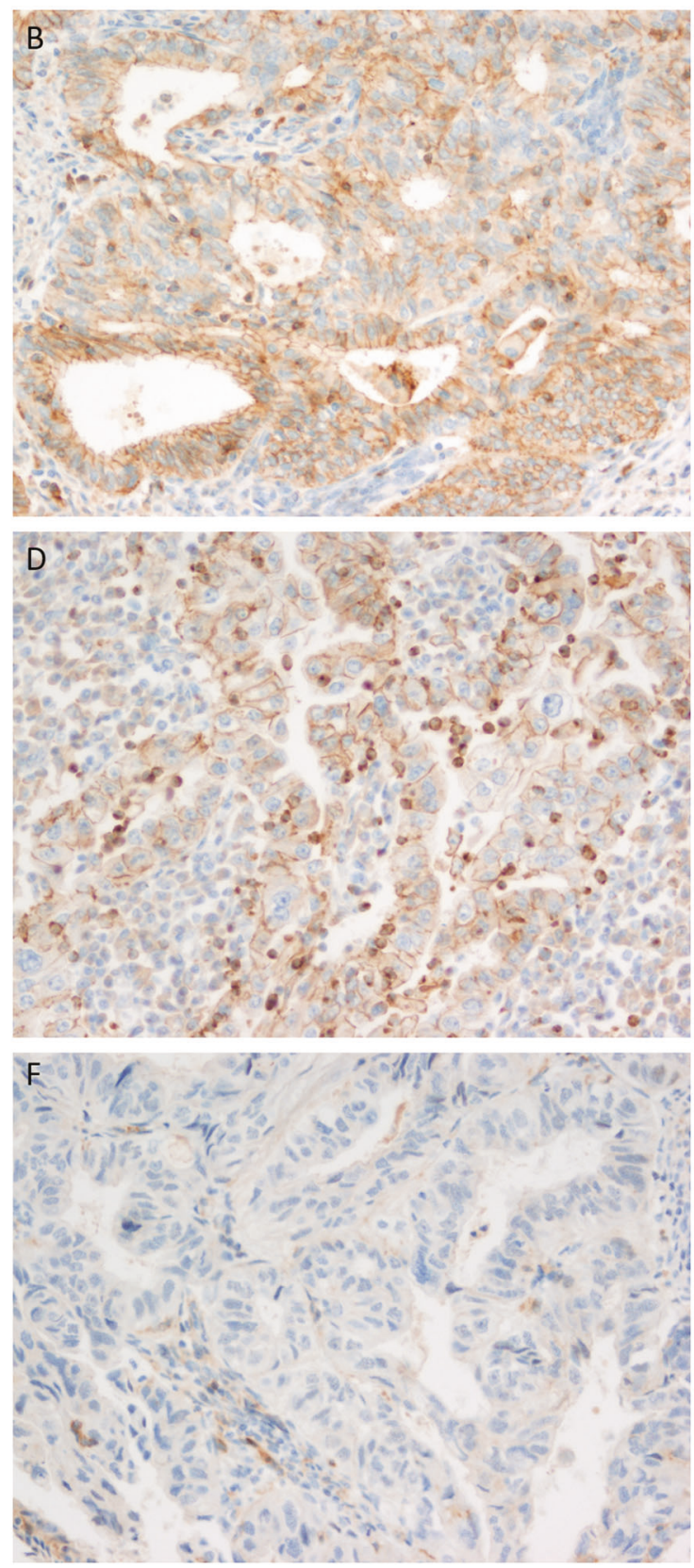

case illustrated in $(\mathbf{c}, \mathbf{d})$ demonstrated patchy tumoral positivity with a more robust TIM-3 positive immune cell infiltrate. In contrast, the mismatch repair-intact endometrial cancer shown in (e, f) was entirely negative for TIM-3 within the tumor cells and showed only rare positive associated lymphocytes

demonstrating other immunohistochemical loss patterns showed TIM-3 staining in at least a subset of cases, with 50\% (2/4) of PMS2-deficient cases and 67\% (8/12) of cases with dual MSH2/MSH6 loss demonstrating $\geq 1 \%$ TIM-3 positivity.

\section{TIM-3 combined positive score}

Using the combined positive score, which accounts for both tumor cell and tumor-associated immune cell 
Table 2 Tumoral + TIM-3 $(\geq 1 \%)$ by grade and mismatch repair status, $n / N(\%)$

\begin{tabular}{|c|c|c|c|c|}
\hline & MMR-Intact & nm-MMRd & MLH1-hm & Total \\
\hline \multirow[t]{7}{*}{ Low-grade (FIGO 1 endometrioid) } & $5 / 9(56 \%)$ & $8 / 13(62 \%)$ & $6 / 8(75 \%)$ & $19 / 30(63 \%)$ \\
\hline & Extent of expression: & Extent of expression: & Extent of expression: & Extent of expression: \\
\hline & $1-5 \%: 4 / 9(44 \%)$ & $1-5 \%: 7 / 13(54 \%)$ & $1-5 \%: 1 / 8(13 \%)$ & $1-5 \%: 12 / 30(40 \%)$ \\
\hline & $6-10 \%: 1 / 9(11 \%)$ & $6-10 \%: 0 / 13(0 \%)$ & $6-10 \%: 2 / 8(25 \%)$ & $6-10 \%: 3 / 30(10 \%)$ \\
\hline & 11-25\%:0/9 (0\%) & $11-25 \%: 0 / 13(0 \%)$ & $11-25 \%: 2 / 8(25 \%)$ & $11-25 \%: 2 / 30(7 \%)$ \\
\hline & $2650 \%: 0 / 9(0 \%)$ & $26-50 \%: 0 / 13(0 \%)$ & $26-50 \%: 0 / 8(0 \%)$ & $26-50 \%: 0 / 30(0 \%)$ \\
\hline & $>50 \%: 0 / 9(0 \%)$ & $>50 \%: 1 / 13(8 \%)$ & $>50 \%: 1 / 8(13 \%)$ & $>50 \%: 2 / 30(7 \%)$ \\
\hline \multirow[t]{7}{*}{ Intermediate-grade (FIGO 2 endometrioid) } & $5 / 8(63 \%)$ & $3 / 3(100 \%)$ & $9 / 10(90 \%)$ & $17 / 21(81 \%)$ \\
\hline & Extent of expression: & Extent of expression: & Extent of expression: & Extent of expression: \\
\hline & $1-5 \%: 4 / 8(50 \%)$ & $1-5 \%: 1 / 3(33 \%)$ & $1-5 \%: 2 / 10(20 \%)$ & $1-5 \%: 7 / 21(33 \%)$ \\
\hline & $6-10 \%: 0 / 8(0 \%)$ & $6-10 \%: 1 / 3(33 \%)$ & $6-10 \%: 5 / 10(50 \%)$ & $6-10 \%: 6 / 21(29 \%)$ \\
\hline & $11-25 \%: 1 / 8(13 \%)$ & $11-25 \%:(0 \%)$ & $11-25 \%: 0 / 10(0 \%)$ & $11-25 \%: 1 / 21(5 \%)$ \\
\hline & $26-50 \%: 0 / 8(0 \%)$ & $26-50 \%:(0 \%)$ & $26-50 \%: 2 / 10(20 \%)$ & $26-50 \%: 2 / 21(10 \%)$ \\
\hline & $>50 \%: 0 / 8(0 \%)$ & $>50 \%: 1 / 3(33 \%)$ & $>50 \%: 0 / 10(0 \%)$ & $>50 \%: 1 / 21(5 \%)$ \\
\hline \multirow{7}{*}{$\begin{array}{l}\text { High-grade (FIGO } 3 \text { endometrioid, dedifferentiated, } \\
\text { and carcinosarcoma) }\end{array}$} & $8 / 8(100 \%)$ & $8 / 9(89 \%)$ & $6 / 7(86 \%)$ & $22 / 24(92 \%)$ \\
\hline & Extent of expression: & Extent of expression: & Extent of expression: & Extent of expression: \\
\hline & $1-5 \%: 3 / 8(38 \%)$ & $1-5 \%: 3 / 9(33 \%)$ & $1-5 \%: 2 / 7(29 \%)$ & $1-5 \%: 8 / 24(33 \%)$ \\
\hline & $6-10 \%: 1 / 8(13 \%)$ & $6-10 \%: 4 / 9(44 \%)$ & $6-10 \%: 1 / 7(14 \%)$ & $6-10 \%: 6 / 24(25 \%)$ \\
\hline & $11-25 \%: 1 / 8(13 \%)$ & $11-25 \%: 0 / 9(0 \%)$ & $11-25 \%: 2 / 7(29 \%)$ & $11-25 \%: 3 / 24(13 \%)$ \\
\hline & $26-50 \%: 1 / 8(13 \%)$ & $26-50 \%: 1 / 9(11 \%)$ & $26-50 \%: 1 / 7(14 \%)$ & $26-50 \%: 3 / 24(13 \%)$ \\
\hline & $>50 \%: 2 / 8(25 \%)$ & $>50 \%: 0 / 9(0 \%)$ & $>50 \%: 0 / 7(0 \%)$ & $>50 \% 2 / 24:(8 \%)$ \\
\hline
\end{tabular}

$M M R$ mismatch repair, $M M R d$ mismatch repair-deficient, $n m$ non-methylated, $h m$ hypermethylated

(both macrophage and lymphocyte) expression, all cases of endometrial carcinoma across mismatch repair subgroups met the threshold of combined positive score $\geq 1$.

\section{Discussion}

Mismatch repair-deficient cancers can show excellent response to immunotherapy targeting the PD-1/PD-L1 immune checkpoint axis, prompting the recent Food and Drug Administration approval of the PD-1 inhibitor Pembrolizumab in solid tumors with this molecular signature [1]. Although the most robust data exists for colorectal carcinomas [1, 4, 43], impressive responses have also been observed in mismatch repair-deficient endometrial cancers $[1,44]$. However, as has repeatedly been demonstrated in other tumor types [14, 16, 17], benefit from anti-PD-1/PDL1 monotherapy has not been uniform. This may be attributable, in part, to the complexity of the immune microenvironment and frequent co-existence of other mechanisms of immune evasion. In addition to expressing high levels of PD-L1, mismatch repair-deficient endometrial carcinomas - particularly Lynch syndrome-associated cancers [8]also often show widespread expression of other immune inhibitory molecules, including the immune checkpoint
CTLA-4 and the enzyme IDO-1 [6, 10, 45]. A recent abstract from Ramos et al. [45] also demonstrated high levels of TIM-3 in mismatch repair-deficient endometrial carcinomas.

Our current study further investigates expression of the immune inhibitory checkpoint molecule TIM-3 in mismatch repair-intact and deficient endometrial carcinomas, with specific attention to non-methylated mismatch repairdeficient vs. MLH1-hypermethylated tumors as well as high-grade, mismatch repair-intact cancers. Our results reveal increased tumor-associated immune cell TIM-3 expression among mismatch repair-deficient cancers when compared to intact cases, which echoes the results from work on PD-L1 [7, 8]. Varying levels of tumoral TIM-3 expression were also observed, with the majority of all cancers displaying at least focal tumoral staining and some cases from each mismatch repair subgroup-including several high-grade intact cancers-demonstrating diffuse expression. Indeed, tumor grade appears to play a more significant role for TIM-3 than it does for PD-L1 [8], with significantly higher expression among intermediate- and high-grade endometrial cancers when compared to lowgrade tumors irrespective of mismatch repair status. TIM-3 expression was not, however, entirely restricted to highgrade cases: two cases with $>50 \%$ expression were lowgrade endometrioid carcinomas. 

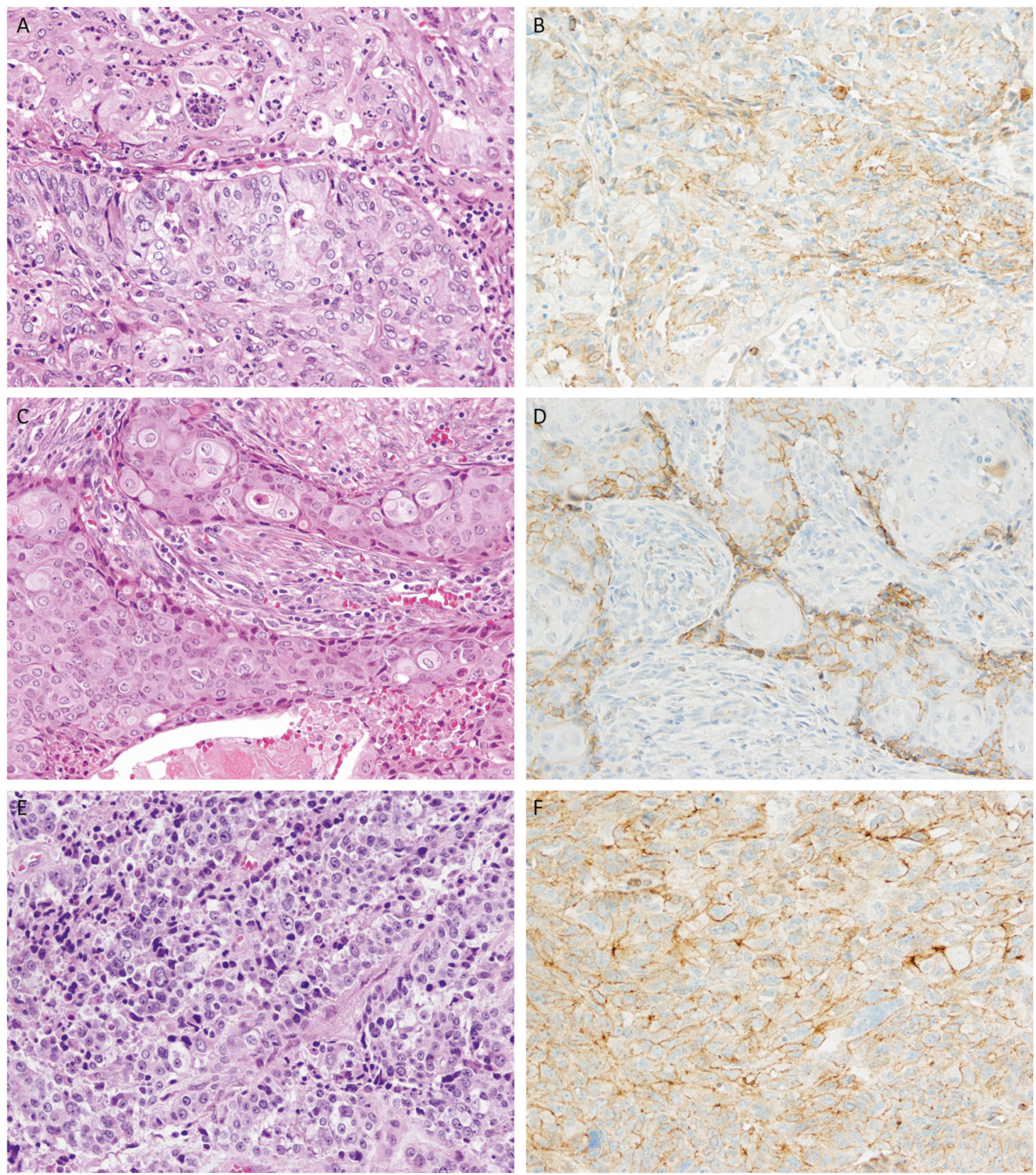

Fig. 3 TIM-3 in high-grade endometrial carcinomas. Tumoral TIM-3 expression was overall more common among intermediate and highgrade cancers when compared to low-grade tumors, with the most dramatic trend observed in the mismatch repair-intact subgroup. The dedifferentiated grade $3 \mathrm{MLH} 1$-hypermethylated cancer depicted in (a, b) showed patchy positivity concentrated in areas of solid architecture,

Although mismatch repair-deficient cancers were not more likely to show $\geq 1 \%$ tumoral TIM-3 expression when compared to intact cases, at the 5\% threshold MLHIhypermethylated cancers were more likely than both mismatch repair-intact and non-methylated mismatch repairdeficient malignancies to be TIM-3 positive. It is important to note, however, that although high-grade cancers were evenly represented across all three mismatch repair subsets

while the MSH6-deficient dedifferentiated tumor in (c, d) showed tumoral expression concentrated at the tumor-stroma interface. The mismatch repair-intact carcinosarcoma illustrated in $(\mathbf{e}, \mathbf{f})$ was among the most strongly positive cases in the series, with regions of widespread expression concentrated in the poorly-differentiated epithelial components of the tumor

and statistically significant differences in tumor grade were not identified across these groups, there were more intermediate-grade carcinomas among the $M L H 1$-hypermethylated and mismatch repair-intact groups when compared to non-methylated mismatch repair-deficient cancers (which were more often low-grade). Thus, in this small sample set, we cannot exclude the possibility that grade contributed to the increased expression observed in the 
Fig. 4 Tumoral TIM-3

expression by histologic grade.

Tumor cell expression of TIM-3

was more common in

intermediate- and high-grade carcinomas when compared to low-grade carcinomas (orange arcs $[<1 \%$, negative]; blue arcs $[\geq 1 \%$, positive])
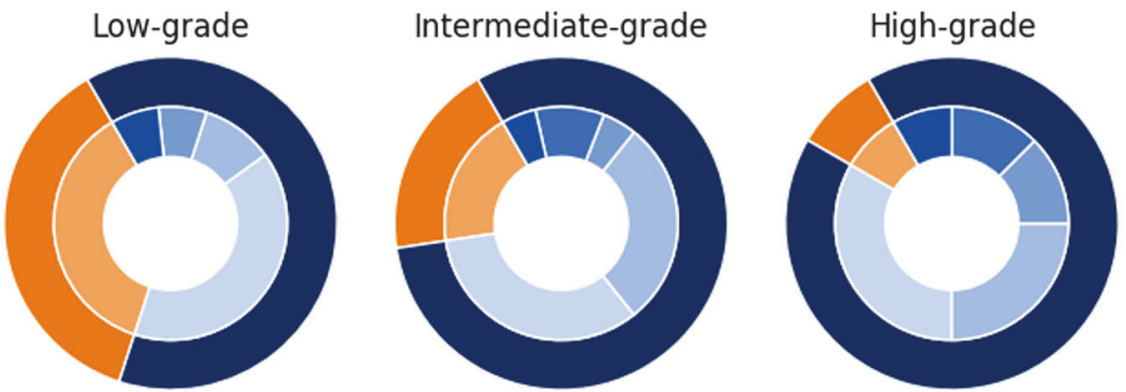

\begin{tabular}{|l|}
\hline$<1 \%(\mathrm{Neg})$ \\
$>1 \%(\mathrm{Pos})$ \\
\hline$<1 \%$ \\
$1-5 \%$ \\
$6-10 \%$ \\
$11-25 \%$ \\
$26-50 \%$ \\
$>50 \%$ \\
\hline
\end{tabular}
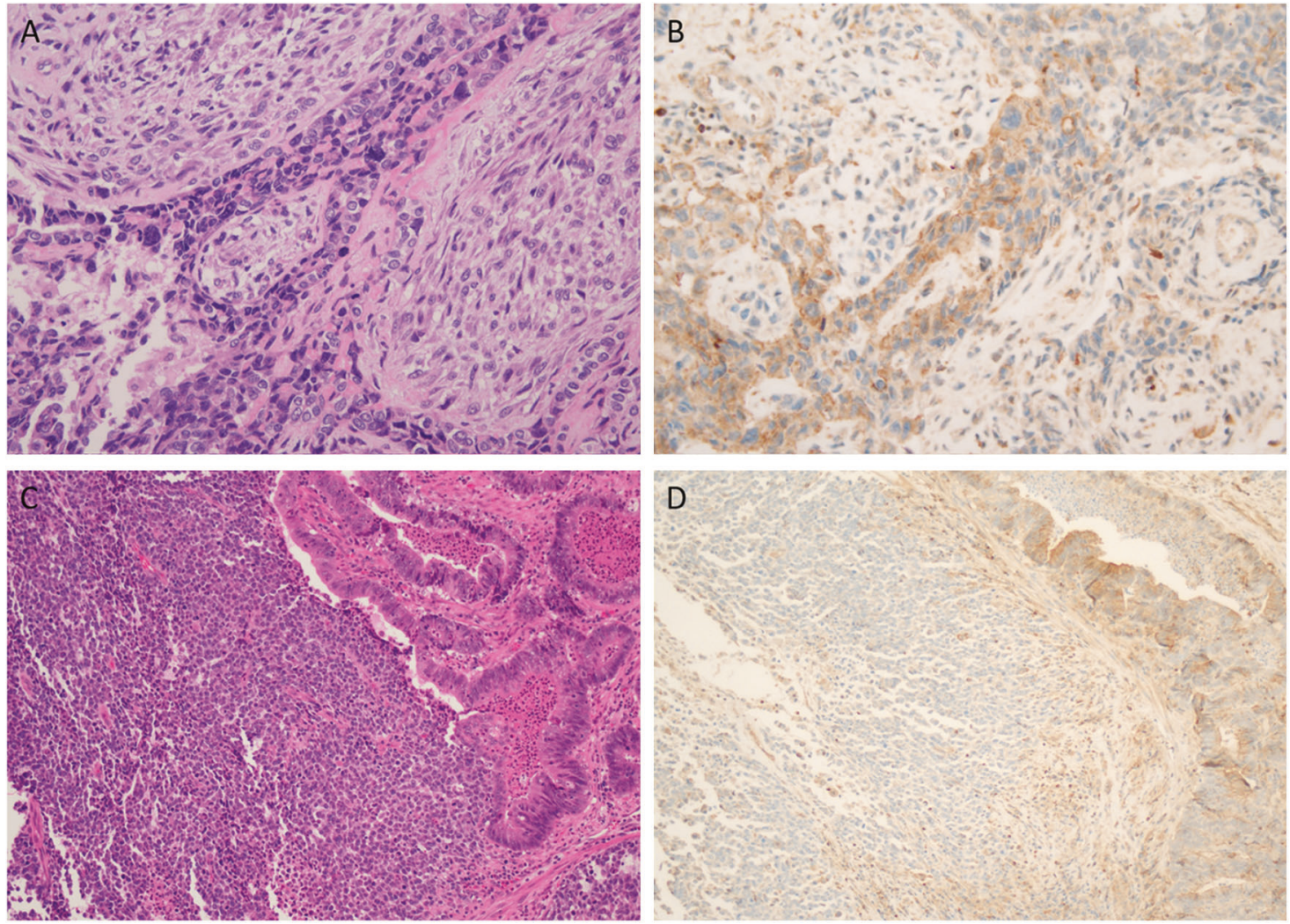

Fig. 5 TIM-3 expression in carcinosarcomas and de-differentiated carcinomas. Both carcinosarcomas in the series demonstrated TIM-3 expression predominantly in the epithelial component of the tumor, as is illustrated in case (a, b). De-differentiated carcinomas, in contrast, showed variable expression patterns, with some showing positivity in

MLH1-hypermethylated group when compared to the nonmethylated mismatch repair-deficient group. Alternatively, it may be that the mechanism of mismatch repair deficiency both well-defined glands and poorly-differentiated elements, and others, like the tumor depicted in (c, d), showing little expression in the poorly-differentiated component but patchy expression in the adjacent well-formed glands

impacts tumoral methods of immune evasion and that MLH1-hypermethylation in particular incites TIM-3 expression. 
Table 3 Tumoral TIM-3 expression and mismatch repair loss patterns in non-methylated mismatch-repair deficient carcinomas, $n / N(\%)$

+TIM-3 $(\geq 1 \%)$ in tumor cells

\begin{tabular}{lc}
\hline MSH6 loss & $8 / 8(100 \%)$ \\
MSH6/MLH1/PMS2 loss & $1 / 1(100 \%)$ \\
MSH2/MSH6 Dual loss & $8 / 12(67 \%)$ \\
PMS2 loss & $2 / 4(50 \%)$
\end{tabular}

A potential role for specific molecular alterations was also observed among the non-methylated mismatch repair-deficient cases. Within this subset, isolated MSH6 loss was most consistently associated with tumoral TIM-3 expression, which parallels what has been observed for PD-L1 [8]. In addition, dramatic response to anti-PD-1 therapy has been noted in an endometrial carcinoma from a patient bearing a germline MSH6 mutation [5]. This is particularly relevant for endometrial carcinomas because MSH6 accounts for a larger proportion of germline mutations when compared to colorectal carcinomas, which predominantly have mutations of $M L H 1$ and $M S H 2$ [46].

These findings underscore the complexity of the endometrial carcinoma tumor microenvironment, and suggest that even among mismatch repair-deficient cancers the ideal targeted immunotherapeutic approach may vary based upon the mechanism of mismatch repair impairment, with cases showing epigenetic MLH1-hypermethylation and MSH6 deficiency perhaps representing particularly good candidates for TIM-3 inhibition. Furthermore, they highlight the potential role for TIM-3 targeting not only mismatch repairdeficient cases, but also a subset of high-grade mismatch repair-intact malignancies.

This is not the first evidence that a proportion of mismatch repair-intact endometrial cancers might also respond to checkpoint inhibition. Early results from the Keynote 028 study have shown some benefit in mismatch repairintact endometrial carcinomas with the PD-1 inhibitor pembrolizumab, with a tumor expression requirement of only $\geq 1 \%$ [18]. Investigations of TIM-3 checkpoint inhibition are more nascent, however: several early phase clinical trials are actively recruiting patients with advanced solid malignancies to assess for potential benefit of targeting this molecule, and results remain forthcoming [clinicaltrials. gov: NCT03489343, NCT02817633, NCT03680508, NCT03652077, NCT02608268].

Such trials, however, do not routinely incorporate biomarker expression in their inclusion criteria. In particular, the clinically meaningful thresholds of expression and the significance of tumoral vs. immune stromal TIM-3 expression have not been established. In many organ systems, only PD-L1 expression in tumor cells is considered predictive of response, and the corollary may be true for TIM-3. However, the recent enlistment of a "combined positive score," which accounts for both immune and tumoral staining, in uterine cervical, gastric/gastroesophageal, and lung small cell carcinoma suggests that focal immune cell expression of inhibitory checkpoint molecules may be sufficient to warrant targeted treatment in some settings [40-42, 47, 48]. In these scenarios, tumors with a combined positive score as low as 1 have garnered FDA-approval for PD-1-based checkpoint inhibitors. In the current series, all cases of endometrial carcinoma across mismatch repair subsets demonstrated a TIM-3 combined positive score of $\geq 1$ due to the ubiquity of at least focal TIM-3-positive immune cells, indicating that the role for TIM-3 inhibition could potentially be widespread in this tumor type. Indeed, prior work has shown that TIM-3positive (TIM-3+/PD-1+/CD8+) lymphocytes have a highly dysfunctional, "exhausted" phenotype that enables continued tumor immune escape [23, 27, 49]. Targeting this subpopulation of lymphocytes has shown promise in reinvigorating the anti-tumor immune response in solid and hematologic cancers [49-52], indicating the importance of considering both the tumoral and immune compartments for the potential success of an immunotherapeutic agent. It remains to be seen, however, whether such limited immune cell expression correlates with improved response to TIM-3 inhibition as it has for PD-L1 and PD-1 inhibitors in some settings [40-42]. Ultimately, determination of the ideal scoring system for TIM-3 will require correlation with expression patterns and outcomes from patients treated with TIM-3 inhibitors, and retrospective analyses of tumor tissue from patients enrolled in current clinical trials will be of interest.

Our findings also highlight the potential role for TIM-3 inhibitors in combination immunotherapy. Tumoral and/or immune expression of TIM-3 may account for resistance to monotherapy with PD-1/PD-L1 agents in some cancers, and presents an opportunity for dual therapy in such cases [20]. Multimodal immunotherapy has been examined with increasing enthusiasm since the success of combination ipilimumab and nivolumab in melanoma [53]. Of even more relevance to this work, the recent CheckMate-142 trial of nivolumab and ipilimumab in mismatch repair-deficient colorectal carcinomas has shown promise and garnered accelerated FDA review [43]. As combination immunotherapy becomes commonplace, standardized approaches to assessing the immune microenvironment as an integrated whole become increasingly relevant. It remains to be seen, however, whether this manifests as an algorithmic series of individual immunohistochemical stains or a single study that utilizes layered fluorescence in situ hybridization or multi-color immunohistochemistry to visualize multiple biomarkers in a single field. 
A significant limitation of this study is the lack of POLE mutation status on mismatch repair-intact cases. Mutations involving POLE are known to generate an ultra-mutated phenotype with increased neoantigen production [7, 54, 55], and one report has demonstrated impressive response to antiPD-1 in an endometrial carcinoma with a POLE mutation [5]. Given that POLE-mutated cases comprise $\sim 10 \%$ of highgrade endometrioid cancers [56,57], we could anticipate that the eight high-grade, mismatch repair-intact cases in this series may include one or more POLE-mutated cancer. While the relative rarity of this mutation makes it unlikely to significantly impact the overall results in this small series, the established relationship between POLE mutations and increased immunogenicity underscores the limitations of mismatch repair status as the sole gatekeeper for immunotherapy access in endometrial carcinomas.

Another important limitation is the fact that many nonmethylated mismatch repair-deficient cases did not have available Lynch syndrome germline testing results, limiting our ability to make definitive conclusions about the specific role of germline-derived mutations in this context. There were also four patients with an immunohistochemical loss pattern suggestive of Lynch syndrome but negative germline testing. Prior studies suggest that these cancers most often demonstrate high level microsatellite instability and bear somatic mismatch repair mutations [58-60], and indeed all four cases in this series were MSI-H. Therefore, the mismatch repair protein loss pattern remains a relatively robust proxy for true mismatch repair-deficiency.

In summary, tumoral and tumor-associated immune cell expression of the immune checkpoint molecule TIM-3 is common among endometrial carcinomas. Although at least focal immune staining was present in all cases, mismatch repair-deficient endometrial carcinomas demonstrated significantly more immune TIM-3 expression than mismatch repair-intact carcinomas. While some tumoral expression was demonstrated across mismatch repair groups, it was most prominent among $M L H 1$-hypermethylated and MSH6-deficient cases. Furthermore, tumoral expression was also observed in some mismatch repair-intact cases, particularly cases with high histologic grade. These results suggest a potential role for TIM-3 checkpoint inhibition among a wide variety of endometrial carcinomas, including some which may not currently be candidates for clinically available immunotherapies.

Acknowledgements The authors would like to thank the University of Virginia Biorepository and Tissue Research Facility for their skill and expertise in performing all immunohistochemical staining.

\section{Compliance with ethical standards}

Conflict of interest The authors declare that they have no conflict of interest.
Publisher's note: Springer Nature remains neutral with regard to jurisdictional claims in published maps and institutional affiliations.

\section{References}

1. Le DT, Durham JN, Smith KN, Wang H, Bartlett BR, Aulakh LK, et al. Mismatch repair deficiency predicts response of solid tumors to PD-1 blockade. Science. 2017;357:409.

2. Lee V, Le DT. Efficacy of PD-1 blockade in tumors with MMR deficiency. Immunotherapy. 2016;8:1-3.

3. Lee V, Murphy A, Le DT, Diaz LA Jr. Mismatch repair deficiency and response to immune checkpoint blockade. Oncologist. 2016;21:1200-11.

4. Le DT, Uram JN, Wang H, Bartlett BR, Kemberling H, Eyring $\mathrm{AD}$, et al. PD-1 blockade in tumors with mismatch-repair deficiency. N Engl J Med. 2015;372:2509-20.

5. Santin AD, Bellone S, Buza N, Choi J, Schwartz PE, Schlessinger J, Lifton RP. Regression of chemotherapy-resistant polymerase E (POLE) ultra-mutated and MSH6 hyper-mutated endometrial tumors with Nivolumab. Clin Cancer Res. 2016;22:5682-7.

6. Liu J, Liu Y, Wang W, Wang C, Che Y. Expression of immune checkpoint molecules in endometrial carcinoma. Exp Ther Med. 2015;10:1947-52.

7. Howitt BE, Shukla SA, Sholl LM, Ritterhouse LL, Watkins JC, Rodig S, et al. Association of polymerase-e-mutated and microsatellite-instable endometrial cancers with neoantigen load, number of tumor-infiltrating lymphocytes, and expression of PD-1 and PD-L1. JAMA Oncol. 2015;1:1319-23.

8. Sloan EA, Ring KL, Willis BC, Modesitt SC, Mills AM. PD-L1 Expression in mismatch repair-deficient endometrial carcinomas, including Lynch syndrome-associated and MLH1 promoter hypermethylated tumors. Am J Surg Pathol. 2017;41:326-33.

9. Vanderstraeten A, Luyten C, Verbist G, Tuyaerts S, Amant F. Mapping the immunosuppressive environment in uterine tumors: implications for immunotherapy. Cancer Immunol Immunother. 2014;63:545-57.

10. Mills A, Zadeh S, Sloan E, Chinn Z, Modesitt SC, Ring KL. Indoleamine 2,3-dioxygenase in endometrial cancer: a targetable mechanism of immune resistance in mismatch repair-deficient and intact endometrial carcinomas. Mod Pathol. 2018;31: 1282-90.

11. Tougeron D, Fauquembergue E, Rouquette A, Le Pessot F, Sesboue R, Laurent M, et al. Tumor-infiltrating lymphocytes in colorectal cancers with microsatellite instability are correlated with the number and spectrum of frameshift mutations. Mod Pathol. 2009;22:1186.

12. Llosa NJ, Cruise M, Tam A, Wicks, EC, Hechenbleikner EM, Taube JM, et al. The vigorous immune microenvironment of microsatellite instable colon cancer is balanced by multiple counterinhibitory checkpoints. Cancer Discov. 2014;5:43-51.

13. Friedman K, Brodsky AS, Lu S, Wood S, Gill AJ, Lombardo K, Yang D, Resnick MB. Medullary carcinoma of the colon: a distinct morphology reveals a distinctive immunoregulatory microenvironment. Mod Pathol. 2016;29:528.

14. Dempke WCM, Fenchel K, Uciechowski P, Dale SP. Second- and third-generation drugs for immuno-oncology treatment-The more the better? Eur J Cancer. 2017;74:55-72.

15. Hellmann MD, Friedman CF, Wolchok JD. Combinatorial cancer immunotherapies. Adv Immunol. 2016;130:251-77.

16. Topalian SL, Hodi FS, Brahmer JR, Gettinger SN, Smith DC, McDermott DF, et al. Safety, activity, and immune correlates of anti-PD-1 antibody in cancer. N Engl J Med. 2012;366:2443-54.

17. Taube JM, Klein A, Brahmer JR, Xu H, Pan X, Kim JH, et al. Association of PD-1, PD-1 ligands, and other features of the 
tumor immune microenvironment with response to anti-PD-1 therapy. Clin Cancer Res. 2014;20:5064-74.

18. Ott PA, Bang Y-J, Berton-Rigaud D, Elez E, Pishvaian MJ, Rugo HS, et al. Pembrolizumab in advanced endometrial cancer: preliminary results from the phase Ib KEYNOTE-028 study. J Clin Oncol. 2016;34:5581-5581.

19. Li X, Hu W, Zheng X, Zheng C, Du P, Zheng Z, et al. Emerging immune checkpoints for cancer therapy. Acta Oncol. 2015;54:1706-13.

20. Koyama S, Akbay EA, Li YY, Herter-Sprie GS, Buczkowski KA, Richards WG, et al. Adaptive resistance to therapeutic PD-1 blockade is associated with upregulation of alternative immune checkpoints. Nat Commun. 2016;7:10501.

21. Nirschl CJ, Drake CG. Molecular pathways: coexpression of immune checkpoint molecules: signaling pathways and implications for cancer immunotherapy. Clin Cancer Res. 2013;19:4917-24.

22. Hughes PE, Caenepeel S, Wu LC. Targeted therapy and checkpoint immunotherapy combinations for the treatment of cancer. Trends Immunol. 2016;37:462-76.

23. Baitsch L, Legat A, Barba L, Fuertes Marraco SA, Rivals JP, Baumgaertner $\mathrm{P}$, et al. Extended co-expression of inhibitory receptors by human $\mathrm{CD} 8 \mathrm{~T}$-cells depending on differentiation, antigen-specificity and anatomical localization. PLoS ONE. 2012;7:e30852.

24. Zhu C, Anderson AC, Schubart A, Xiong H, Imitola J, Khoury SJ, et al. The TIM-3 ligand galectin-9 negatively regulates $\mathrm{T}$ helper type 1 immunity. Nat Immunol. 2005;6:1245.

25. Du W, Yang M, Turner A, Xu C, Ferris RL, Huang J, Kane LP, Lu B. TIM-3 as a target for cancer immunotherapy and mechanisms of action. Int J Mol Sci. 2017;18:645.

26. Sánchez-Fueyo A, Tian J, Picarella D, Domenig C, Zheng XX, Sabatos CC, et al. TIM-3 inhibits T helper type 1-mediated autoand alloimmune responses and promotes immunological tolerance. Nat Immunol. 2003;4:1093.

27. Das M, Zhu C, Kuchroo VK. TIM-3 and its role in regulating antitumor immunity. Immunol Rev. 2017;276:97-111.

28. Rangachari M, Zhu C, Sakuishi K, Xiao S, Karman J, Chen A, et al. Bat 3 promotes $\mathrm{T}$ cell responses and autoimmunity by repressing TIM-3-mediated cell death and exhaustion. Nat Med. 2012;18:1394-1400.

29. Sakuishi K, Jayaraman P, Behar SM, Anderson AC, Kuchroo VK. Emerging TIM-3 functions in antimicrobial and tumor immunity. Trends Immunol. 2011;32:345-9.

30. Dardalhon V, Anderson AC, Karman J, Apetoh L, Chandwaskar R, Lee DH, et al. TIM-3/galectin-9 pathway: regulation of Th1 immunity through promotion of $\mathrm{CD} 11 \mathrm{~b}(+) \mathrm{Ly}-6 \mathrm{G}(+)$ myeloid cells. J Immunol. 2011;185:1383-92.

31. Yan J, Zhang Y, Zhang JP, Liang J, Li L, Zheng L. TIM-3 expression defines regulatory $\mathrm{T}$ cells in human tumors. PLoS ONE Electron Resour. 2013;8:e58006.

32. Cao Y, Zhou X, Huang X, Li Q, Gao L, Jiang L, Huang M, Zhou J. TIM-3 Expression in cervical cancer promotes tumor metastasis. PLoS ONE. 2012;8:e53834.

33. Jiang J, Jin M-S, Kong F, Cao D, Ma HX, Jia Z, Wang YP, Suo J, Cao X. Decreased galectin-9 and increased TIM-3 expression are related to poor prognosis in gastric cancer. PLoS ONE. 2013;8: e81799.

34. Morgado M, Datar I, Wang J, Sanmamed MF, McEachern K, Jenkins D, et al. Abstract 1681: Simultaneous measurement and significance of PD-1, LAG-3 and TIM-3 expression in human solid tumors. Cancer Res. 2018;78:1681.

35. Yuan J, Jiang B, Zhao H, Huang Q. Prognostic implication of TIM-3 in clear cell renal cell carcinoma. Neoplasma. 2014;61:3540.

36. Sloan EA, Moskaluk CA, Mills AM. Mucinous differentiation with tumor infiltrating lymphocytes is a feature of sporadically methylated endometrial carcinomas. Int $\mathrm{J}$ Gynecol Pathol. 2017;36:205-16.

37. Mills AM, Longacre TA. Lynch syndrome screening in the gynecologic tract: current state of the art. Am J Surg Pathol. 2016;40:e35-e44.

38. Mills AM, Liou S, Ford JM, Berek JS, Pai RK, Longacre TA. Lynch syndrome screening should be considered for all patients with newly diagnosed endometrial cancer. Am J Surg Pathol. 2014;38:1501-9.

39. Büttner R, Gosney JR, Skov BG, Adam J, Motoi N, Bloom KJ, et al. Programmed death-ligand 1 immunohistochemistry testing: a review of analytical assays and clinical implementation in nonsmall-cell lung cancer. J Clin Oncol. 2017;35:3867-76.

40. Kulangara K, Zhang N, Corigliano E, Guerrero L, Waldroup S, Jaiswal D, et al. Clinical utility of the combined positive score for programmed death ligand-1 expression and the approval of Pembrolizumab for treatment of gastric cancer. Arch Pathol Lab Med. 2018. https://doi.org/10.5858/arpa.2018-0043-OA.

41. Salhab M, Migdady Y, Donahue M, Xiong Y, Dresser K, Walsh W, Chen BJ, Liebmann J. Immunohistochemical expression and prognostic value of PD-L1 in extrapulmonary small cell carcinoma: a single institution experience. $\mathrm{J}$ Immunother Cancer. 2018;6:42. -018-0359-1

42. Chung HC, Schellens JHM, Delord J-P, Perets R, Italiano A, Shapira-Fromer R, et al. Pembrolizumab treatment of advanced cervical cancer: Updated results from the phase 2 KEYNOTE-158 study. J Clin Oncol. 2018;36:5522-5522.

43. Overman MJ, Lonardi S, Wong KYM, Lenz HJ, Gelsomino F, Aglietta M, et al. Durable clinical benefit with Nivolumab plus Ipilimumab in DNA mismatch repair-deficient/microsatellite instability-high metastatic colorectal cancer. J Clin Oncol. 2018;36:773-9.

44. Fader AN, Diaz LA, Armstrong DK, Tanner EJ, Uram J, Eyring A, et al. Preliminary results of a phase II study: PD-1 blockade in mismatch repair-deficient, recurrent or persistent endometrial cancer. Gynecol Oncol. 2016;141:206-7.

45. Ramos A, Fortin SAM, Melchert V, Jenkins D, Borger DR, Growdon WB. Checkpoint inhibitor signatures across endometrial carcinoma histologic subtypes. Gynecol Oncol. 2018;149:621.

46. Bonadona V, Bonaïti B, Olschwang S, Grandjouan S, Huiart L, Longy $\mathrm{M}$, et al. Cancer risks associated with germline mutations in MLH1, MSH2, and MSH6 genes in lynch syndrome. JAMA. 2011;305:2304-10.

47. Fashoyin-Aje L, Donoghue M, Chen H, He K, Veerarghavan J, Goldberg KB, et al. FDA Approval Summary: Pembrolizumab for recurrent locally advanced or metastatic gastric or gastroesophageal junction adenocarcinoma expressing PD-L1. Oncologist. 2019;24:103-9.

48. Frenel J-S, Le Tourneau C, O'Neil B, Ott PA, Piha-Paul SA, Gomez-Roca C, et al. Safety and efficacy of pembrolizumab in advanced, programmed death ligand 1-positive cervical cancer: results from the phase Ib KEYNOTE-028 trial. J Clin Oncol. 2017;35:4035-41.

49. Sakuishi K, Apetoh L, Sullivan JM, Blazar BR, Kuchroo VK, Anderson AC. Targeting TIM-3 and PD-1 pathways to reverse T cell exhaustion and restore anti-tumor immunity. J Exp Med. 2010;207:2187-94.

50. Fourcade J, Sun Z, Benallaoua M, Guillaume P, Luescher IF, Sander C, et al. Upregulation of TIM-3 and PD-1 expression is associated with tumor antigen-specific CD8(+) T cell dysfunction in melanoma patients. J Exp Med. 2010;207:2175-86.

51. Zhou Q, Munger ME, Veenstra RG, Weigel BJ, Hirashima M, Munn DH, et al. Coexpression of TIM-3 and PD-1 identifies a CD8 + T-cell exhaustion phenotype in mice with disseminated acute myelogenous leukemia. Blood. 2011;117:4501-10. 
52. Ngiow SF, Teng MWL, Smyth MJ. Prospects for TIM3-targeted antitumor immunotherapy. Cancer Res. 2011;71:6567-71.

53. Wolchok JD, Kluger H, Callahan MK, Postow MA, Rizvi NA, Lesokhin AM, et al. Nivolumab plus Ipilimumab in advanced melanoma. N Engl J Med. 2013;369:122-33.

54. Kandoth C, McLellan MD, Vandin F, Ye K, Niu B, Lu C, et al. Mutational landscape and significance across 12 major cancer types. Nature. 2013;502:333.

55. Talhouk A, McConechy MK, Leung S, Li-Chang HH, Kwon JS, Melnyk N, et al. A clinically applicable molecular-based classification for endometrial cancers. Br J Cancer. 2015;113:299.

56. Meng B, Hoang LN, McIntyre JB, Duggan MA, Nelson GS, Lee $\mathrm{CH}$, Köbel M. POLE exonuclease domain mutation predicts long progression-free survival in grade 3 endometrioid carcinoma of the endometrium. Gynecol Oncol. 2014;134:15-19.

57. Bosse T, Nout RA, McAlpine JN, McConechy MK, Britton H, Huessein YR, et al. Molecular classification of grade 3 endometrioid endometrial cancers identifies distinct prognostic subgroups. Am J Surg Pathol. 2018;42:561-8.

58. Mills AM, Sloan EA, Thomas M, Modesitt SC, Stoler MH, Atkins KA, Moskaluk CA. Clinicopathologic comparison of Lynch syndrome-associated and "Lynch-like" endometrial carcinomas identified on universal screening using mismatch repair protein immunohistochemistry. Am J Surg Pathol. 2016;40: 155-65.

59. Haraldsdottir S, Hampel H, Tomsic J, Frankel WL, Pearlman R, de la Chapelle A, Pritchard CC. Colon and endometrial cancers with mismatch repair deficiency can arise from somatic, rather than germline, mutations. Gastroenterology. 2014;147:1308-16.

60. Watkins JC, EJ Yang, Muto MG, Feltmate CM, Berkowitz RS, Horowitz NS, et al. Universal screening for mismatch-repair deficiency in endometrial cancers to identify patients with Lynch syndrome and Lynch-like syndrome. Int $\mathbf{J}$ Gynecol Pathol. 2017;36:115-27. 